\title{
Human History Is Convergent History
}

\author{
Nguyen Ba Trinh \\ Vietnam Academy of Science and Technology, Vietnam
}

\begin{abstract}
ABSTRAT
As is known, in nature and human society, there are often laws that are common or similar. However, human society is a very complex system. Therefore, sociologists often take ideas from natural sciences as a research model. This method was used by René Descartes, Herbert Spencer, Kenneth D. Bailey, Talcott Parson, Niklas Luhmann, Erich Jantsh, and Peter Grimes.

In the scale of the universe, matter has evolved along the trend of convergence. Thanks to that trend, the first stars were formed, followed by galaxies, black holes and heavy chemical elements. The trend of convergence also exists in the water environment on Earth and leads to the formation of life. Biochemical molecules also increase the degree of convergence in biological evolution.

The trend of convergence in material evolution has provided us with a glimpse into human society. In fact, human society has also evolved along the trend of convergence. In prehistoric times, people lived scattered in groups and later formed clans. From tribes, convergence led to the formation of tribes, from tribes led to tribal alliances and emirates, then to nations. Entering the era of civilization, the speed of convergence is increasing, it is reflected in the convergence of urbanization and formation of national alliances, such as the EU (European Union), ASEAN (Association of Countries Southeast Asia) AU (African Union), UNASUR (Union of South American Nations).

There are also many other forms of coalitions between countries, such as the International Trade Organization (WTO), the Asia-Pacific Forum (APEC) ... At the same time, in different countries on Political institutions or ideologies are tending to converge to similar points. Thus convergence is a general law of evolution of nature and society.

Currently, humanity has to join hands to solve many problems, such as the environment, terrorism, disease problems ... Therefore, social convergence is not only a law but also an urgent need. Due to science, technology and international trade are growing, human knowledge and culture are expanding, so people are more connected with each other. Therefore, human history is a convergence history.
\end{abstract}

Keywords: Convergence, human history, evolution. 